más importantes como lo son el manufacturero, el comercio al por mayor y menor, entre otros. Naudé y Szirmai (2012) afirman que los países en vía de desarrollo deben tener entre sus prioridades atender el sector manufacturero para que el desarrollo del mismo origine empleo de alta calidad, y que los productos no solo se consuman localmente sino que tengan oportunidad de ser exportados.

En Ecuador las MIPYMES de la industria manufacturera tienen dos fortalezas que son: capacidad de adaptación y generación de empleo (Aguiar et al., 2013). El presente estudio se ha realizado para conocer el entorno en el que se desarrollan MIPYMES del sector manufacturero en Ecuador en los años 2014-2015 para lo cual se utiliza el enfoque Estructura-Conducta-Resultado (ECR) (Bain, 1951) que consiste en analizar los elementos principales que determinan la estructura de un mercado y su relación con la conducta y el desempeño de las empresas.

Para inferir la estructura del mercado es necesario primero definir el mercado relevante de las empresas en estudio, para esto se utilizó la desagregación del nivel 2 del código CIIU ya que en éste se considera los productos sustitutos cercanos. De aquí se puede determinar la estructura del mercado mediante índices de concentración que muestran cómo se distribuye la participación del mercado entre las distintas empresas que lo componen, los que se utilizan en su mayoría son el ratio de concentración (CR4 y CR8) que mide las ventas acumuladas de las 4 y 8 mayores empresas de la industria respectivamente en relación con las ventas totales del sector en análisis, y el índice Herfindahl-Hirschman (IHH) que es la suma de los cuadrados de las participaciones de todas las empresas del mercado. (Tarziján y Paredes, 2006).

Evaluar cuantitativamente la concentración del mercado ayuda no solo a establecer una relación entre la estructura del mercado y el desempeño de las empresas, sino también a desarrollar políticas públicas que las autoridades gubernamentales encargadas de la regulación del mercado antimonopolio puedan crear (Krivka, 2016). Por esto el objetivo del presente artículo es determinar el ambiente competitivo en el que operan las MIPYMES ecuatorianas a través del análisis de la concentración del mercado manufacturero en los años 2014-2015 y así poder sugerir el establecimiento de políticas públicas que regulen este importante sector económico. Se debe agregar que durante la revisión de la literatura se encontró que existen algunas investigaciones que utilizan el enfoque ECR, sin embargo analizan otros sectores diferentes al manufacturero por lo que estudios para este sector son escasos a nivel regional y de Ecuador.

La competencia en los diferentes sectores de una economía ayuda a aumentar el bienestar social ya que reduce precios y elimina los excedentes del productor, además genera mejoras en los productos o servicios proporcionados a los consumidores. Las MIPYMES en Ecuador podrían encontrarse compitiendo en los diferentes actividades de la industria manufacturera de una manera desigual, esto ocasionado por cierto poder de mercado de las empresas más grandes o inclusive por prácticas colusivas y/o barreras a la competencia, de tal manera que esto pueda afectar a su vez la permanencia en el tiempo de las MIPYMES en un determinado mercado.

La estructura del estudio será el siguiente: La sección 2 presenta la revisión de la literatura, la sección 3 revisa la metodología y materiales de la investigación, la sección 4 muestra los resultados y discusión de los mismos, por último la sección 5 las conclusiones.

\section{Revisión de la literatura}

El sector manufacturero es uno de los sectores principales en algunas economías mundiales ya que estimula a transformar la estructura de economías tradicionales a economías modernas además, incrementa la productividad. (Naudé y Szirmai, 2012).

Kaldor (1975) afirma cuatro leyes de crecimiento económico que son: 1) La tasa de crecimiento de la economía se relaciona con la del sector manufacturero. 2) Un incremento de la tasa de crecimiento de la manufactura genera un aumento de la productividad en el mismo sector. 3) La productividad de los sectores ajenos a la manufactura aumenta cuando el producto manufacturero se incrementa y 4) Factores de oferta y demanda inciden en las diferentes tasas de crecimiento de la producción manufacturera.

Es así que en Ecuador el sector con mayor peso en el PIB en el 2014 es la manufactura representando el 11,68\%, mientras que en el 2015 este porcentaje descendió ligeramente a 11,57\% (Banco Central del Ecuador, 2016). En la figura 1 se presenta la clasificación de la industria manufacturera por actividades y su respectiva participación en el PIB de esta industria. Según el INEC la producción de manufactura en el 2014 fue de $\$ 28^{\prime} 093.000$.

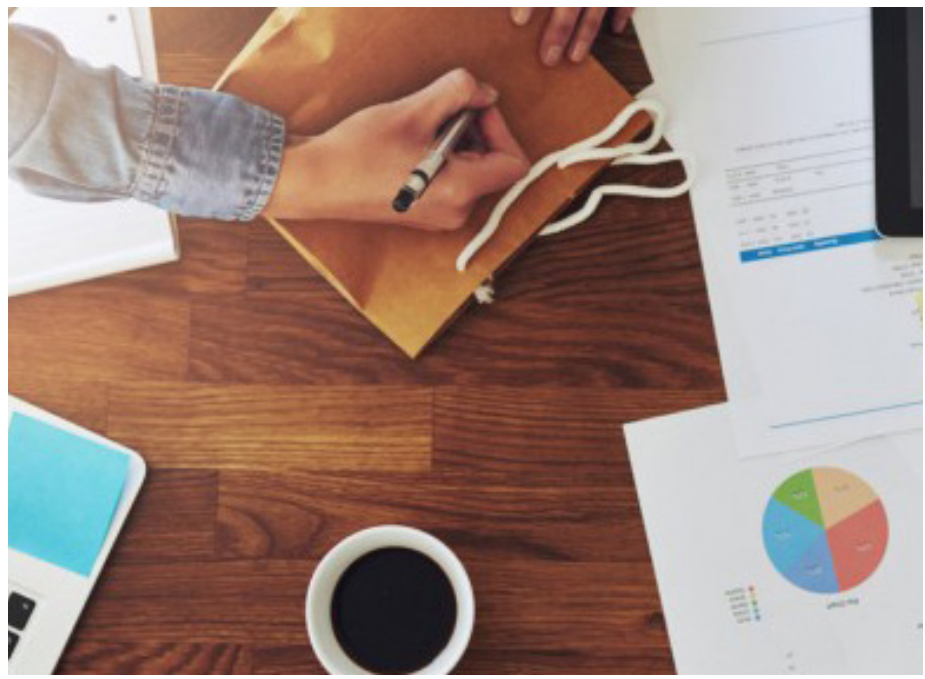


Figura 1. Composición del PIB manufacturero por actividades

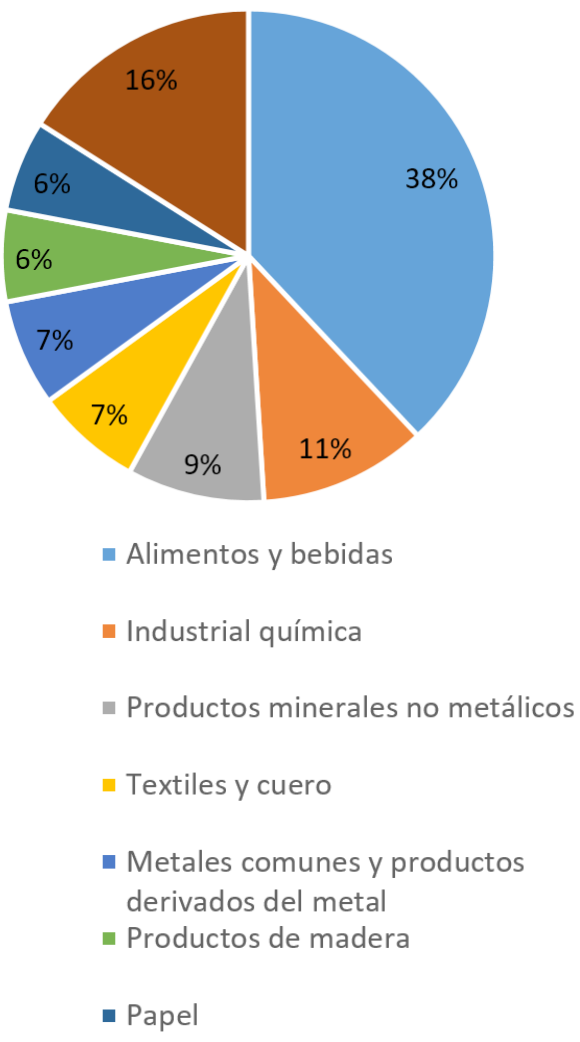

Fuente: Banco Central del Ecuador.

Bain (1954) demostró que las empresas con mayor grado de concentración, tenían en promedio ganancias más altas, es decir se encuentran correlacionadas positivamente, a esta relación la denominó paradigma Estructura-ConductaResultado (ECR) (Thong, 2012).

El paradigma ECR se estableció con el objetivo de analizar el entorno en el que operan las empresas (Mohamed, Shamsudin, Latif y Mu’azu, 2013), fundamentalmente se plantea qué características del mercado afecta la rentabilidad de las empresas, como por ejemplo la concentración. Esta teoría tiene sus bases en la estructura de la empresa que se analiza que a mayor concentración del mercado, menor competencia, y además que el desempeño de la empresa está relacionado positivamente con la eficiencia de la estructura (Bassey, Ibok y Akapaeti, 2013).

Una consecuencia positiva posible del paradigma ECR, es hacer de este análisis una guía para la política (Jedlicka y Jumah, 2006), así la política de competencia podrá contribuir a una estructura deseable de la industria y a una política reguladora que intervenga en la conducta de los actores (Njuguna, 2014).

Por el contrario, Claessens (2009) hace una aseveración negativa referente a la inalterable relación causal estructuraconducta-resultado, señala que la estructura se ve afectada por la conducta y resultado de las empresas. Con ventajas y desventajas el paradigma ECR hasta la actualidad sigue siendo uno de los análisis más importantes para investigadores de Organización Industrial (Sheel, 2016).

En la literatura pese a existir varios medios para calcular la concentración de un mercado, los principales índices utilizados son el índice Herfindahl- Hirschman (IHH) y el ratio de concentración $(\mathrm{Cr}(\mathrm{m}))$ por el sencillo cálculo que implican (Krivka, 2016). Por ejemplo en el sector agrícola de Malasia se tuvo como objeto de estudio a la industria de aves, el IHH en el 2009 fue de 0.42 que demuestra que la industria es altamente concentrada en dicho año y por lo tanto indica una estructura oligopolística; los resultados para $\mathrm{Cr} 4$ y $\mathrm{Cr} 8$ fueron $64 \%$ y $74 \%$ respectivamente, manifiestan que es un mercado moderadamente concentrado en las cuatro y ocho empresas con mayor cuota de mercado (Mohamed, Shamsudin, Latif y Mu’azu, 2013). En el sector de servicios financieros en Kenia se encontró un IHH de 0.98 en el periodo 2009-2011 que indica una alta concentración y poder de mercado que está permitido por la regulación estatal de dicho país, debido a la naturaleza irreversible a largo plazo de los productos, la colusión entre administradores de pensiones y los jugadores, la falta de sustitutos cercanos a las anualidades y la ausencia de diferenciación (Njuguna, 2014).

De manera general no se han realizado estudios del sector manufacturero, a nivel regional solo se encontró un estudio al subsector confitería de Cali-Colombia, en el que se evidencia que en el 2010 esta industria estaba altamente concentrada mediante el cálculo del IHH que fue de 0.38 (González y Rosero, 2011).

Vázquez (2015) analizó el sector de alimentos, bebidas y tabaco en México en el periodo de 1994-2008 y encontró que la industria es una de las más importantes de la manufactura mexicana, en el 2008 representó $25.9 \%$ del total de la producción. En un análisis realizado en Rumania se encontró que la industria manufacturera de ese país posee bajo nivel de tecnología y de productividad laboral (Herman, 2016). Por otro lado, Aguiar et al. (2013) realiza un análisis descriptivo al sector manufacturero ecuatoriano en el periodo de 19902008 encontrando un ligero aumento en la productividad de ese sector.

En cuanto a estudios por tamaño de empresa en la literatura se encontró investigaciones sobre las pequeñas y medianas empresas (PYMES) y estas son consideradas como un factor importante porque contribuyen a la economía, en países desarrollados como Turquía representa el 99,9\% de la industria pero solo el $55 \%$ de ellas están operando en sectores de valor añadido (Şener, Savrul y Aydın, 2014).

En el presente estudio se analizará el ambiente competitivo en el que operan las MIPYMES el sector manufacturero de Ecuador, por tal motivo cabe mencionar que en América Latina se estudió la participación de este tipo de empresas, Saavedra y Hernández (2008) encontraron que en promedio 
el 99,12\% de las MIPYMES representan el total de empresas y el 13\% de las mismas pertenecen al sector de la manufactura, además son generadoras de empleo, en promedio en $64,26 \%$ a nivel regional. Mientras que en un estudio más detallado de la generación de empleo de las MIPYMES, hallaron que la generación de empleo es distinta en cada región de México del sector manufacturero. (Acolt y Flores, 2010).

\section{Metodología}

Los índices de concentración se los puede calcular por diferentes grupos, clasificándolos mediante cierto número de las empresas más grandes de la industria, entre los más usuales están el Cr4 y Cr8, los cuales miden la cuota de mercado de las cuatro y las ocho empresas más grandes de la industria respectivamente (Beattie, Goodacre y Fearnley, 2003).

Ratio de Concentración (CRn): Es suma de las cuotas de mercado $\left(\mathrm{s}_{\mathrm{i}}\right)$ de las $(\mathrm{m})$ empresas mayores del sector, por lo tanto es el valor que toma la curva de concentración para el punto (m). (Núñez y Pérez, 2001; Ginevičius y Čirba, 2007; Ginevičius y Cirba, 2009; Ginevičius, Petraškevičius y Šimkūnaitè, 2010).

$$
C R_{m}=\sum_{i=1}^{m} s_{i}
$$

$$
C R_{4}=s_{1}+s_{2}+s_{3}+s_{4}=\frac{q_{1}}{Q}+\frac{q_{2}}{Q}+\frac{q_{3}}{Q}+\frac{q_{4}}{Q}
$$

En donde:

$\mathrm{q}_{\mathrm{i}}=$ ingresos totales de la empresa $\mathrm{i}$.

$\mathrm{Q}=$ ingresos totales de todo el mercado.

Una de las ventajas que posee este ratio es que solo se necesita la información de las (m) empresas más grandes (Hannaford, 2007). Si el índice de concentración es 1 , se asume que la industria se comporta como un monopolio puro o una posición monopolística en términos de cuota de mercado de las empresas de la observación. Los parámetros de lectura del ratio de concentración estipulados por la Superintendencia de Control del Poder de Mercado del Ecuador (2017), son los siguientes:

- $\mathrm{Cr}(\mathrm{m})$ inferior a 0.33 , el mercado no está concentrado.

- $\mathrm{Cr}(\mathrm{m})$ entre 0.33 y 0.67 , el mercado está moderadamente concentrado.

- $\mathrm{Cr}(\mathrm{m})$ mayor a 0.67 , el mercado está altamente concentrado.

Además de conocer la participación acumulada de un número determinado de las empresas más grandes de cada actividad manufacturera se analiza la concentración en base a la población total de empresas, esto se realiza mediante el índice Herfindahl- Hirschman (IHH) ampliamente utilizado en la literatura de organización industrial (Tirole, 1988), el mismo que considera la cuota de mercado de las empresas examinando a todos los miembros de la industria, además es el índice más adecuado para analizar precios de las industrias donde las empresas evaluadas tienen comportamiento competitivo (Church y Ware, 2000; González-Laxe, NovoCorti y Morollón, 2014).

Índice de Herfindahl-Hirschman (IHH): Es la suma de los cuadrados de las cuotas de mercado de todas las empresas de una industria (U.S Department of Justice and the Federal Trade Commission, 2010) y se define como sigue:

$$
\begin{gathered}
H H I=\sum_{i=1} S_{i}^{2} \\
H H I=\left(\frac{q_{1}}{Q}\right)^{2}+\left(\frac{q_{2}}{Q}\right)^{2}+\left(\frac{q_{3}}{Q}\right)^{2}+\cdots+\left(\frac{q_{n}}{Q}\right)^{2}
\end{gathered}
$$

En donde:

$\mathrm{q}_{\mathrm{n}}=$ Ingreso total de la empresa $\mathrm{n}$.

$\mathrm{Q}=$ Ingreso totales de todo el mercado.

$\mathrm{n}=$ Número de empresas de la industria.

Las cuotas de mercado se miden en términos porcentuales, una de las ventajas es que tiene en cuenta a todas las empresas de la industria. U.S. Department of Justice and the Federal Trade Commission (2010) ha definido las siguientes escalas del índice IHH:

- $\mathrm{IHH} \leq 0.10$, el mercado no está concentrado.

- $0.10<\mathrm{IHH} \leq 0.18$, el mercado está moderadamente concentrado.

- IHH >0.18, el mercado está altamente concentrado.

El cálculo del Î́ndice de Herfindahl-Hirschman se lo utiliza también para poder calcular el Número Equivalente, el mismo que muestra el número de empresas de debería tener cada actividad manufacturera si la distribución del sector fuera equitativa (Sánchez y Mogro, 2017; González-Laxe, Novo-Corti y Morollón, 2014).

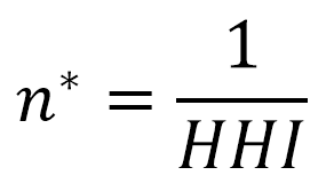

En este análisis se usa el código de Clasificación Industrial Internacional Uniforme (CIIU) dado que agrupa las actividades económicas semejantes por niveles, es decir, agrupa las empresas que poseen similar tecnología y procesos de producción.

Para inferir la estructura del mercado es necesario definir el mercado relevante de las empresas en estudio, para esto se utilizó la desagregación del nivel 2 del código CIIU ya que en éste se considera los productos sustitutos cercanos (Tabla 1). 
Tabla 1. Ramas de la industria manufacturera a dos dígitos del código CIIU

\begin{tabular}{|c|c|}
\hline \begin{tabular}{|c|} 
Código \\
CIIU \\
\end{tabular} & Actividad empresarial \\
\hline $\mathrm{C}$ & Industria Manufacturera \\
\hline $\mathrm{C} 10$ & Elaboración de productos alimenticios \\
\hline $\mathrm{C} 11$ & Elaboración de bebidas \\
\hline $\mathrm{C} 12$ & Elaboración de productos de tabaco \\
\hline $\mathrm{C} 13$ & Elaboración de productos textiles \\
\hline $\mathrm{C} 14$ & Elaboración de prendas de vestir \\
\hline $\mathrm{C} 15$ & Elaboración de cueros y productos conexos \\
\hline $\mathrm{C} 16$ & $\begin{array}{l}\text { Producción de madera y fabricación de } \\
\text { productos de madera y corcho, excepto } \\
\text { muebles; fabricación de artículos de paja y } \\
\text { de materiales trenzables }\end{array}$ \\
\hline $\mathrm{C} 17$ & Fabricación de papel y productos de papel \\
\hline $\mathrm{C} 18$ & Impresión y reproducción de grabaciones \\
\hline C19 & $\begin{array}{l}\text { Fabricación de coque y de productos de la } \\
\text { refinación del petróleo }\end{array}$ \\
\hline $\mathrm{C} 20$ & $\begin{array}{l}\text { Fabricación de substancias y productos } \\
\text { químicos }\end{array}$ \\
\hline $\mathrm{C} 21$ & $\begin{array}{l}\text { Fabricación de productos farmacéuticos, } \\
\text { sustancias químicas medicinales y produc- } \\
\text { tos botánicos de uso farmacéutico }\end{array}$ \\
\hline $\mathrm{C} 22$ & $\begin{array}{l}\text { Fabricación de productos de caucho y } \\
\text { plástico }\end{array}$ \\
\hline C23 & $\begin{array}{l}\text { Fabricación de otros productos minerales } \\
\text { no metálicos }\end{array}$ \\
\hline $\mathrm{C} 24$ & Fabricación de metales comunes \\
\hline $\mathrm{C} 25$ & $\begin{array}{l}\text { Fabricación de productos elaborados de } \\
\text { metal, excepto maquinaria y equipo }\end{array}$ \\
\hline $\mathrm{C} 26$ & $\begin{array}{l}\text { Fabricación de productos de informática, } \\
\text { electrónica y óptica }\end{array}$ \\
\hline $\mathrm{C} 27$ & Fabricación de equipo eléctrico \\
\hline $\mathrm{C} 28$ & Fabricación de maquinaria y equipo N.C.P \\
\hline $\mathrm{C} 29$ & $\begin{array}{l}\text { Fabricación de vehículos automotores, } \\
\text { remolques y semirremolques }\end{array}$ \\
\hline $\mathrm{C} 30$ & $\begin{array}{l}\text { Fabricación de otros tipos de equipos de } \\
\text { transporte }\end{array}$ \\
\hline $\mathrm{C} 31$ & Fabricación de muebles \\
\hline $\mathrm{C} 32$ & Otras industrias manufactureras \\
\hline $\mathrm{C} 33$ & $\begin{array}{l}\text { Reparación e instalación de maquinaria y } \\
\text { equipo }\end{array}$ \\
\hline
\end{tabular}

Fuente: INEC.
Se realizó el análisis a las actividades de la industria manufacturera en Ecuador. En Latinoamérica no existe una resolución para definir las MIPYMES, sin embargo los países que pertenecen a la Comunidad Andina de Naciones (2009), (CAN) -Bolivia, Colombia, Ecuador, Perú- clasifican a las MIPYMES según tres criterios: número de trabajadores, total ingresos y activos de las empresas; estos criterios lo aplican también los países latinoamericanos que no pertenecen a la CAN (Comunidad Andina de Naciones, 2009). En Ecuador se consideran los dos principales criterios para definir a las MIPYMES - número de trabajadores y total ingresos- (Tabla 2) sin embargo, el presente estudio considera el criterio de Total Ingresos para el estudio de los índices de concentración, este criterio se registra en el formulario 101 del Servicio de Rentas Internas (SRI).

Tabla 2. Definición de las MIPYMES en Ecuador.

\begin{tabular}{|l|c|c|r|r|}
\hline $\begin{array}{c}\text { Tamaño } \\
\text { de la } \\
\text { empresa }\end{array}$ & \multicolumn{2}{|c|}{$\begin{array}{c}\text { Número de } \\
\text { trabajadores } \\
\text { Mínimo }\end{array}$} & \multicolumn{2}{c|}{ Total Ingresos } \\
\hline Micro & 1 & 9 & \multicolumn{1}{c|}{ Mínimo } & \multicolumn{1}{c|}{ Máximo } \\
\hline Pequeña & 10 & 49 & $\$ 100,001.00$ & $\$ 100,000.00$ \\
\hline Mediana & 50 & 199 & $\$ 1,000,001.00$ & $\$ 5,000,000.000$ \\
\hline
\end{tabular}

Fuente: Art. 106. Reglamento a la estructura e institucional de desarrollo productivo.

\section{Datos}

Se utiliza la base de datos de los estados financieros por rama económica de los años 2014 y 2015 obtenida de la página web de la Superintendencia de Compañías, Valores y Seguros (2016), en la que se encuentra 3.345 y 3.138 MIPYMES respectivamente en los años mencionados, no se consideró las empresas con ingreso nulo o negativo.

Las MIPYMES del sector manufacturero de Ecuador representan entre todas las empresas - incluyendo las grandes para el año 2014 y 2015 el $84.77 \%$ y $84.72 \%$ respectivamente. En la figura 2 se muestra el porcentaje de empresas MIPYMES y grandes para cada actividad de la industria manufacturera para los años 2014 y 2015. Las MIPYMES poseen más del $50 \%$ de participación en todas las actividades manufactureras de esta industria.

En promedio el mayor porcentaje de participación que alcanzan las empresas grandes es el $20 \%$, así se cumple en las actividades elaboración de productos alimenticios (C10), fabricación de papel y productos de papel (C17), fabricación de coque y productos de refinación del petróleo (C19), fabricación de productos farmacéuticos, sustancias químicas medicinales y productos botánicos de uso farmacéutico (C21), fabricación de productos de caucho y plástico (C22), fabricación de metales comunes (C24) y fabricación de vehículos automotores, remolques y semirremolques (C29). La única actividad que se encuentra perfectamente distribuida es elaboración de productos de tabaco (C12) ya que posee dos empresas MIPYMES y dos empresas grandes. 
Figura 2. Relación de MIPYMES y grandes empresas del nivel 2 del código CIIU 2014-2015

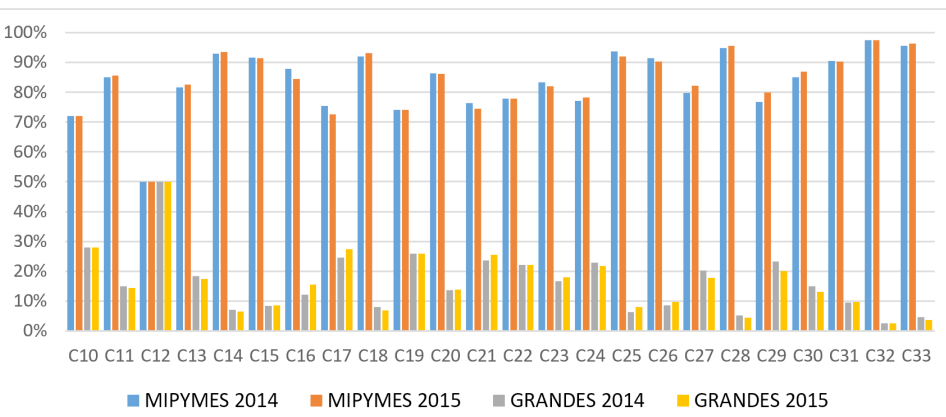

La tabla 3 muestra los montos mínimos y máximos de ingresos totales según el tamaño de empresa en los años 2014 y 2015, de tal manera que se pueda observar las diferentes brechas de ingresos entre cada grupo. Así, la empresa mediana que obtuvo mayor ingreso es 4.5 veces más grande en ingresos que la empresa pequeña que obtuvo más ingresos y 45.5 veces más que la microempresa con mayores ingresos.

Tabla 3: Ingresos mínimos y máximos de las micro, pequeñas y medianas empresas

\begin{tabular}{|l|r|r|}
\hline 2014 & \multicolumn{1}{|c|}{ Mínimo } & \multicolumn{1}{c|}{ Máximo } \\
\hline Micro & $\$ 0.15$ & $\$ 99,865.80$ \\
\hline Pequeña & $\$ 100,092.15$ & $\$ 999,449.39$ \\
\hline Mediana & $\$ 1,050,074.85$ & $\$ 4,549,451.82$ \\
\hline
\end{tabular}

\begin{tabular}{|l|r|r|}
\hline $\mathbf{2 0 1 5}$ & \multicolumn{1}{|c|}{ Mínimo } & \multicolumn{1}{c|}{ Máximo } \\
\hline Micro & $\$ 0.01$ & $\$ 99,844.50$ \\
\hline Pequeña & $\$ 101,032.24$ & $\$ 999,922.58$ \\
\hline Mediana & $\$ 1,002,292.07$ & $\$ 4,991,319.26$ \\
\hline
\end{tabular}

\section{Resultados y Discusión}

En la tabla 4 se muestra el ratio de concentración Cr4, Cr8, IHH y número equivalente de cada actividad objeto de estudio de la industria manufacturera para el año 2014, en la columna resultado se encuentra el nivel de concentración de cada actividad manufacturera según el IHH.

De todas las actividades analizadas, la única en la que se evidencia un monopolio puro mediante el índice $\mathrm{Cr} 4$ es elaboración de productos de tabaco (C12) dado que el total de empresas que opera en esta actividad son exactamente cuatro, además este resultado se confirma mediante el cálculo del IHH ya que muestra que este sector se encuentra altamente concentrado, por otro lado cuando se calcula el número equivalente el resultado es 2 , lo que significaría que este mercado opera como si existiesen tan solo dos empresas con una misma cuota de mercado.

Elaboración de bebidas (C11), producción de madera y fabricación de productos de madera y corcho (C16), fabricación de coque y de productos de refinación del petróleo
(C19), fabricación de productos de informática, electrónica y óptica (C26) y fabricación de vehículos automotores, remolques y semirremolques (C29) son las actividades en las que las cuatro y las ocho empresas más grandes de ese sector tienen la mayor cantidad de cuota de mercado y por encima de 0,67 , es decir, existe una alta concentración.

Estos resultados se complementan con el índice de IHH, el cual se presenta en un mayor valor en las actividades mencionadas sobre estas líneas excepto en $\mathrm{C} 16$, un $\mathrm{IHH}$ mayor a 0.18 muestra que la distribución de las cuotas de mercado es desigual entre las empresas que forman parte de las actividades mencionadas, lo que refleja indirectamente la existencia de líderes de mercado o con una posición dominante dando como resultado que estas actividades estén altamente concentradas, es decir estas empresas se desarrollan en un entorno menos competitivo. Mientras que 11 de las 24 actividades de la industria manufacturera en el 2014 se desenvuelven en un ambiente competitivo, así lo indica el resultado del IHH en las actividades que se las denomina no concentradas.

Por otro lado, existe una relación negativa entre el tamaño de los sectores y su grado de concentración, es decir las actividades de mayor tamaño empresarial no están concentradas mientras que, las actividades de menor tamaño están altamente concentradas. Por ejemplo, en las figuras 3 y 4 las actividades que predominan en tamaño son elaboración de productos alimenticios $(\mathrm{C} 10)$ y reparación e instalación de maquinaria y equipo (C33) las mismas que no están concentradas. Por otro lado, entre las actividades de menor tamaño están elaboración de productos de tabaco (C12) y fabricación de coque y de productos de refinación del petróleo (C19), y estas actividades son algunas de las que están altamente concentradas. Por lo que el postulado que pocas y grandes empresas (alta concentración) son más fáciles de comprometerse en conductas anticompetitivas (colusión) se podría estar cumpliendo.

Además se analizó el cálculo del número equivalente, que muestra la distribución de un sector de empresas con cuotas de mercado equitativamente distribuidos, es decir su resultado es cercano a cuantas empresas operarían con igual participación sobre los ingresos. Un número más pequeño mostraría un mercado concentrado y un número más grande mostraría un mercado más competitivo. Los resultados de este índice coinciden con los del IHH y el CR4, ya que las mismas actividades denominadas altamente concentradas muestran números inferiores al 4. 
Figura 3. Número de empresas por actividad 2014

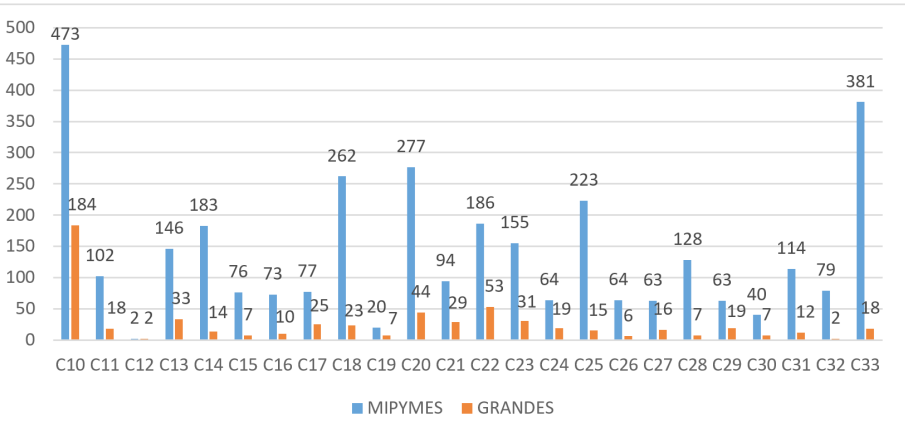

Tabla 4. Resultado de índices año 2014

\begin{tabular}{|r|r|r|r|r|l|}
\hline CIIU & $\begin{array}{c}\text { Índice } \\
\text { Cr4 }\end{array}$ & $\begin{array}{c}\text { Índice } \\
\text { Cr8 }\end{array}$ & IHH & $\begin{array}{c}\text { Número } \\
\text { quiva- } \\
\text { lente }\end{array}$ & \multicolumn{1}{|c|}{ Resultado } \\
\hline C10 & 0.25 & 0.35 & 0.03 & 33 & No concentrado \\
\hline C11 & 0.88 & 0.92 & 0.24 & 4 & $\begin{array}{l}\text { Altamente } \\
\text { concentrado }\end{array}$ \\
\hline C12 & 1 & N/A & 0.58 & 2 & $\begin{array}{l}\text { Altamente } \\
\text { concentrado }\end{array}$ \\
\hline C13 & 0.24 & 0.35 & 0.03 & 33 & No concentrado \\
\hline C14 & 0.39 & 0.51 & 0.07 & 14 & No concentrado \\
\hline C15 & 0.37 & 0.53 & 0.05 & 20 & No concentrado \\
\hline C16 & 0.69 & 0.81 & 0.18 & 6 & $\begin{array}{l}\text { Moderadamente } \\
\text { concentrado }\end{array}$ \\
\hline C17 & 0.41 & 0.7 & 0.07 & 14 & No concentrado \\
\hline C18 & 0.3 & 0.46 & 0.04 & 25 & No concentrado \\
\hline C19 & 0.8 & 0.91 & 0.27 & 4 & $\begin{array}{l}\text { Altamente } \\
\text { concentrado }\end{array}$ \\
\hline C20 & 0.41 & 0.57 & 0.06 & 17 & No concentrado \\
\hline C21 & 0.49 & 0.67 & 0.1 & 10 & No concentrado \\
\hline C22 & 0.3 & 0.44 & 0.04 & 25 & No concentrado \\
\hline C23 & 0.58 & 0.72 & 0.15 & 7 & $\begin{array}{l}\text { Moderadamente } \\
\text { concentrado }\end{array}$ \\
\hline C24 & 0.67 & 0.85 & 0.13 & 8 & $\begin{array}{l}\text { Moderadamente } \\
\text { concentrado }\end{array}$ \\
\hline C25 & 0.51 & 0.62 & 0.08 & 13 & No concentrado \\
\hline C26 & 0.81 & 0.89 & 0.44 & 2 & $\begin{array}{l}\text { Altamente } \\
\text { concentrado }\end{array}$ \\
\hline C27 & 0.49 & 0.67 & 0.09 & 11 & No concentrado \\
\hline C28 & 0.52 & 0.64 & 0.15 & 7 & $\begin{array}{l}\text { Moderadamente } \\
\text { concentrado }\end{array}$ \\
\hline C29 & 0.83 & 0.88 & 0.32 & 3 & $\begin{array}{l}\text { Altamente } \\
\text { concentrado }\end{array}$ \\
\hline C30 & 0.63 & 0.86 & 0.12 & 8 & $\begin{array}{l}\text { Moderadamente } \\
\text { concentrado }\end{array}$ \\
\hline
\end{tabular}

Fuente: Superintendencia de Compañías, Valores y Seguros.

Respecto al año 2015, en la tabla 5 se evidencia un ligero incremento de la concentración de las actividades de las cuales fabricación de productos de informática, electrónica y óptica (C26) es la actividad que más aumentó su concentración tanto en las cuatro como en las ocho mayores empresas de este sector, el índice de Cr4 incrementó 6 puntos porcentuales y el índice $\mathrm{Cr} 8$ aumentó 5 puntos porcentuales, por lo cual esta actividad sigue perteneciendo al grupo de las actividades altamente concentradas es decir, esta actividad tiene poca competencia mostrando así que el poder de mercado puede ser sostenido en el tiempo.

El resultado del índice IHH 2015 es similar al del 2014 para las actividades de la industria manufactura, excepto para fabricación de equipo eléctrico (C27) ya que pasa del estado de actividad no concentrada a actividad moderadamente concentrada es decir, en 2014 había más competencia que en el 2015 para esta actividad. 
Figura 4. Número de empresas por actividad 2015

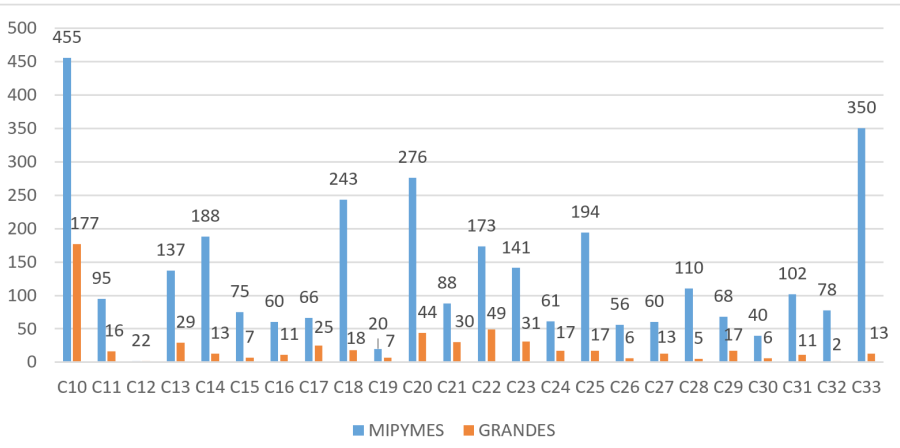

Tabla 5. Resultado de índices año 2015

\begin{tabular}{|l|c|c|c|c|l|}
\hline CIIU & $\begin{array}{c}\text { Índice } \\
\text { Cr4 }\end{array}$ & $\begin{array}{c}\text { Índice } \\
\text { Cr8 }\end{array}$ & IHH & $\begin{array}{c}\text { Número } \\
\text { equiva- } \\
\text { lente }\end{array}$ & \multicolumn{1}{|c|}{ Resultado } \\
\hline C10 & 0.26 & 0.37 & 0.03 & 33 & No concentrado \\
\hline C11 & 0.88 & 0.93 & 0.24 & 4 & $\begin{array}{l}\text { Altamente } \\
\text { concentrado }\end{array}$ \\
\hline C12 & 1 & N/A & 0.63 & 2 & $\begin{array}{l}\text { Altamente } \\
\text { concentrado }\end{array}$ \\
\hline C13 & 0.21 & 0.33 & 0.02 & 50 & No concentrado \\
\hline C14 & 0.4 & 0.53 & 0.08 & 13 & No concentrado \\
\hline C15 & 0.37 & 0.55 & 0.05 & 20 & No concentrado \\
\hline C16 & 0.66 & 0.81 & 0.16 & 6 & $\begin{array}{l}\text { Moderadamente } \\
\text { concentrado }\end{array}$ \\
\hline C17 & 0.41 & 0.7 & 0.07 & 14 & No concentrado \\
\hline C18 & 0.33 & 0.49 & 0.04 & 25 & No concentrado \\
\hline C19 & 0.83 & 0.94 & 0.32 & 3 & $\begin{array}{l}\text { Altamente } \\
\text { concentrado }\end{array}$ \\
\hline C20 & 0.4 & 0.55 & 0.06 & 17 & No concentrado \\
\hline C21 & 0.51 & 0.67 & 0.10 & 10 & No concentrado \\
\hline C22 & 0.29 & 0.44 & 0.04 & 25 & No concentrado \\
\hline C23 & 0.57 & 0.73 & 0.13 & 8 & $\begin{array}{l}\text { Moderadamente } \\
\text { concentrado }\end{array}$ \\
\hline C24 & 0.65 & 0.85 & 0.13 & 8 & $\begin{array}{l}\text { Moderadamente } \\
\text { concentrado }\end{array}$ \\
\hline C25 & 0.52 & 0.65 & 0.08 & 13 & No concentrado \\
\hline C26 & 0.87 & 0.94 & 0.54 & 2 & $\begin{array}{l}\text { Altamente } \\
\text { concentrado }\end{array}$ \\
\hline C27 & 0.56 & 0.76 & 0.11 & 9 & $\begin{array}{l}\text { Moderadamente } \\
\text { concentrado }\end{array}$ \\
\hline C28 & 0.49 & 0.6 & 0.14 & 7 & $\begin{array}{l}\text { Moderadamente } \\
\text { concentrado }\end{array}$ \\
\hline C29 & 0.8 & 0.87 & 0.31 & 3 & $\begin{array}{l}\text { Altamente } \\
\text { concentrado }\end{array}$ \\
\hline C30 & 0.66 & 0.83 & 0.12 & 8 & $\begin{array}{l}\text { Moderadamente } \\
\text { concentrado }\end{array}$ \\
\hline C31 & 0.59 & 0.71 & 0.11 & 9 & $\begin{array}{l}\text { Moderadamente } \\
\text { concentrado }\end{array}$ \\
\hline
\end{tabular}

Fuente: Superintendencia de Compañías, Valores y Seguros.
Los resultados encontrados de las actividades: elaboración de productos alimenticios (C10), elaboración de prendas de vestir (C14) y fabricación de productos farmacéuticos, sustancias químicas medicinales y productos botánicos de uso farmacéutico (21) indican que las empresas pertenecientes a estas actividades se desarrollan en mercados no concentrados es decir, en un ambiente competitivo, estos resultados difieren con el estudio de Horna, Osorio y Guachamín, (2009) en el que analizaron la concentración del sector manufacturero de Ecuador mediante el código CIIU a 3 dígitos desde el año 2000 al 2008 ya que en ese periodo estas actividades fueron moderadamente concentrados.

Mientras que fabricación de otros productos minerales no metálicos (C23) y fabricación de metales comunes en este estudio se presentaron como actividades moderadamente concentradas, coincidiendo estos resultados con el estudio de Horna, Osorio y Guachamín (2009). Cabe indicar que el trabajo con el que se ha comparado resultados es el único de análisis al sector manufacturero ecuatoriano que se encontró durante la revisión de la literatura.

El presente trabajo posee ciertas limitaciones que específicamente vienen dadas por la base de datos empleada, ya que puede existir variaciones en los resultados dado que se utiliza la base de datos de la Superintendencia de Compañías, Valores y Seguros (2016) y ésta es frecuentemente actualizada al existir empresas que no presentan los balances financieros hasta las fechas establecidas y otras que presentan sus estados financieros de manera atrasada, lo cual implica una afectación directa en la base de datos.

\section{Conclusiones}

El sector manufacturero en el Ecuador ha sido uno de los sectores más importantes por su aportación al Valor Añadido Bruto (VAB) y por su participación dentro del PIB, esto es uno de los motivos principales para analizar qué es lo que ocurre dentro de cada uno de sus actividades a nivel CIIU dos dígitos.

Las MIPYMES en Ecuador juegan un rol importante dentro de los diferentes mercados, tanto así que para el sector manufacturero representan el $84.77 \%$ y $84.72 \%$ para los años 2014 y 2015 del total de empresas que tienen ingresos totales positivos. Esto evidencia que una aplicación de políticas públicas que afecten a las MIPYMES tendría un impacto directo en la actividad empresarial tanto para la generación de empleo como para el incremento de la riqueza y el dinamismo económico.

En este estudio se ha calculado los índices de concentración más utilizados según la literatura para determinar el ambiente competitivo en el que operan las empresas, todo esto siguiendo la metodología propuesta a partir del paradigma ECR, donde en primer lugar se analiza la estructura del sector manufacturero a dos dígitos del CIIU de tal manera que 
conociendo el nivel de ingresos totales de cada empresa de cada actividad se ha obtenido las participaciones de mercado que poseen cada una, una vez hallado sus cuotas de mercado se ha analizado su comportamiento a partir del CR4, CR8, IHH y número equivalente y a partir de estos indicadores se han obtenido resultados del ambiente competitivo en el que operan las MIPYMES del sector manufacturero.

Para el año 2014 y 2015, cinco actividades manufactureras son consideradas altamente concentradas según los indicadores de concentración analizados, justamente en esas cinco actividades es donde menor cantidad de MIPYMES operan en el sector manufacturero, algo contrario ocurre en las actividades no concentradas, en donde existen una mayor cantidad de MIPYMES.

La relación obtenida muestra una posible dificultad para las MIPYMES de operar en mercados altamente concentrados ya que estos mercados se encuentran estructurados por pocas empresas grandes con una participación de mercado muy alta haciendo así que tengan cierto poder de mercado sobre aquellas empresas con poca participación y además de eso que pudieran realizar prácticas anticompetitivas como la colusión o aplicar barreras de entrada a nuevos competidores. El problema del poder de mercado en una economía como la ecuatoriana es que podría causar inconvenientes no solo en las MIPYMES sino también problemas sociales, como la posibilidad de disminuir el bienestar a través del aumento de precios de ventas y de esta forma poder aumentar la rentabilidad de pocas empresas; problemas de productividad, al disminuir la eficiencia y la eficacia de la elaboración de productos; problemas de crecimiento económico ya que podrían impedir la creación de nuevas empresas por las barreras de entrada y por la difícil situación de mantenerse en el tiempo de las empresas.

Uno de los principales problemas en mercados altamente concentrados y donde las empresas podrían ejercer poder de mercado es que es duradero, es decir se mantiene en el tiempo (Baker, 2017). Y estas empresas dominantes podrían mantener esa hegemonía mediante la aplicación de barreras de entrada a MIPYMES, generando así destrucción de empresas y a su vez de empleo. Sin duda, muchas actividades económicas son consideradas oligopolios competitivos (concentración moderada) para lo cual es importante una correcta regulación a tiempo para evitar que se conviertan en actividades altamente concentradas o en monopolios.

Por lo que, al determinar mercados altamente $\mathrm{y}$ moderadamente concentrados los Gobiernos deberían aplicar regulaciones a esas actividades buscando evitar las prácticas anticompetitivas pero sobretodo buscando aumentar la competencia empresarial ya que eso ayudaría a la creación de empresas y a la generación de empleo; las empresas buscarían innovar y ser más productivas para poder obtener mayores cuotas de mercado y tener mejor rentabilidad.
Las MIPYMES diariamente deben enfrentarse a problemas comunes de la competencia como las guerras de precios, la diversificación, la búsqueda de nuevos compradores, mercados y hasta la supervivencia, por esto los entes encargados deberían aplicar regulaciones para que la disputa se vuelva menos compleja, de tal manera que se busque evitar las fusiones y adquisiciones entre empresas que al generarse una sola lleguen a ocupar el $30 \%$ de la cuota total del mercado como se explica según la Ley Orgánica del Control de Poder de Mercado de Ecuador (2011); además que se generen leyes para que en el caso del sector cervecero (parte de la actividad C11) en Ecuador donde una empresa tiene el $98 \%$ del mercado se regule de tal manera que impulsen el aumento de cuota de mercado a empresas más pequeñas (cervezas artesanales), así también como tener ciertos canales de ventas para que se mantengan en el tiempo; podrían aplicarse multas a las prácticas de barreras de entradas a nuevas empresas como una posible disminución de precios hasta el costo medio total, la apoderación del excedente del consumidor y las prácticas de exclusividad de productos en ciertas zonas geográficas y/o canales de distribución.

Este estudio pretende impulsar futuras investigaciones en análisis de concentración de mercado ya no solo a nivel del código CIIU a dos dígitos, sino también a 6 dígitos por actividad económica y además utilizar otras herramientas o indicadores de concentración con el que se puedan contrastar los utilizados en este estudio. Un análisis econométrico es viable a medida que se obtenga una mayor información para estimar índices de Lerner o Panzar y Rosse.

\section{Listado de Referencias}

Acolt, R. y Flores, M. (2010). Desempeño regional de la Micro, Pequeña y Mediana Empresa del sector manufacturero. Investigación y Ciencia, 18(47), 31-38.

Aguiar, V., Arghoty, A., Burgos, S., Gualavisí, M., Onofa, M., Ruiz, P., y Zambrano, R. (2013). Estudios industriales de la micro, pequeña y mediana empresa. FLACSO Sede Ecuador, Ecuador.

Banco Central del Ecuador, (2016). Boletín de Cuentas Nacionales trimestrales $N^{\circ}$ 97. Disponible en: https://www.bce.fin.ec/index.php/ component/k2/item/763 [Revisado 9 de Dic. 2016].

Bain, J. S. (1951). Relation of profit rate to industry concentration: American manufacturing, 1936-1940. The Quarterly Journal of Economics, 293-324.

Bain, J. (1954). Economies of scale, concentration, and the condition of entry in twenty manufacturing industries. The American Economic Review, 15-39. Bassey, N. E., Ibok, O. W., y Akapaeti, A. J. (2013). Rice Market Structure, Conduct and Performance in Nigeria: A Survey of Akwa Ibom State Rice Marketers. Asian Journal of Agriculture and Food Science (ISSN: 23211571), 1(03).

Baker, J. B. (2017). Market power in the U.S. economy today. Washington Center for Equitable Growth.

Beattie, V., Goodacre, A., y Fearnley, S. (2003). And then there were four: A study of UK audit market concentration-causes, consequences and the scope for market adjustment. Journal of Financial Regulation and Compliance, 11(3), 250-265.

Comunidad Andina de Naciones (2009). Disposición Técnica para la transmisión de datos de estadísticas de PYME de los países miembros de la Comunidad Andina, Pub. L. No. 1260. 
Church J. $y$ Ware, R. (2000): Industrial Organization: A Strategic Approach. McGrawHill

Claessens, S. (2009). Competition in the Financial Sector: Overview of Competition Policies. Working paper WP/09/45, IMF.

Herman, E. (2016). The Importance of the Manufacturing Sector in the Romanian Economy. Procedia Technology, 22, 976 - 983.

Ginevičius, R., y Čirba, S. (2007). Determining market concentration. Journal of Business Economics and Management, 8(1), 3-10.

Ginevičius, R., y Cirba, S. (2009). Additive measurement of market concentration. Journal of Business Economics and Management, 10(3), 191198.

Ginevičius, R., Petraškevičius, V., y Šimkūnaitè, J. (2010). The influence of market concentration on the results of enterprise commercial activities. Business: Theory and Practice/Verslas: Teorija ir Praktika, 11(3), 185-193. González, J., y Rosero, M. (2011). Análisis de estructura, conducta y desempeño del subsector de la confitería en el área metropolitana de Cali 2002-2010, vol. 7, 2, 72-84. Universidad Libre Cali, Colombia.

González-Laxe, F., Novo-Corti, I., y Morollón, F. R. (2014). Análisis de la concentración, la especialización y el liderazgo de los puertos españoles: un estudio de los efectos de la crisis a los puertos españoles. In International conference on regional science: Financing the role of the regions and towns in economic recovery.

Hannaford, S. (2007). Market domination!: The impact of industry consolidation on competition, innovation, and consumer choice. Greenwood Publishing Group.

Horna, L., Osorio, N., y Guachamín, M. (2009). Análisis de mercado del sector industrias manufactureras en base a CIIU 3 bajo un enfoque de concentración económica en el período 2000-2008 en el Ecuador. Revista Politécnica. Vol. 30(1): 230-243.

Instituto Nacional de Estadisticas y Censos (INEC) (2014). Clasificación Nacional de Actividades Económicas.

Jedlicka, L., y Jumah, A. (2006). The Austrian Insurance Industry: A structure, conduct and performance analysis (No. 189). Institute for Advanced Studies. Kaldor, N. (1975). Economic Growth and the Verdoorn Law--A Comment on Mr Rowthorn's Article. The Economic Journal. 85(340), 891-896.

Krivka, A. (2016). On the concept of market concentration, the minimum Herfindahl-Hirschman index, and its practical application. Panoeconomicus. 63(5), 525-540.

Ley Orgánica del Control de Poder de Mercado de Ecuador (2011). Control del Poder del Mercado. Registro Oficial, Suplemento, 555, 13.
Mohamed, Z., Shamsudin, M. N., Latif, I. A., y Mu'azu, A. U. (2013). Measuring competition along the supply chain of the Malaysian poultry industry. In International Conference on Social Science Research (pp. 4-5).

Naudé W., y Szirmai, A., (2012). The importance of manufacturing in economic development: Past, present and future perspectives. UNU-MERIT Working Papers.

Njuguna, A. G. (2014). Competition in the Financial Services Sector: A Case of Kenyan Annuities Market. International Journal of Business and Social Research. 4(10), 75-91.

Núñez, S., y Pérez, M. (2001). El grado de concentración en las ramas productivas de la economía española (No. 0113). Banco de España.

Saavedra, G. M. L., y Hernández, Y. (2008). Caracterización e importancia de las MIPYMES en Latinoamérica: Un estudio comparativo. Actualidad contable faces, 11(17), 122-134.

Sánchez, C. U., y Mogro, S. C. (2017). Estructura de la competencia del sector tabacalero en España: cigarrillos y tabaco de liar. Ciencia Unemi, 10(22), 20-28.

Şener, S., Savrul, M., y Aydin, O. (2014). Structure of Small and MediumSized Enterprises in Turkey and Global Competitiveness Strategies. ProcediaSocial and Behavioral Sciences. 150, 212-221.

Sheel, A. (2016). SCP-relevance and class-effect in performance-A comparative analysis of restaurants and petroleum firms. International Journal of Hospitality Management. 52, 33-45.

Superintendencia de Compañias, Valores y Seguros del Ecuador (2016). Portal de Información de la Superintendencia de Compañias, Valores y Seguros del Ecuador.

Superintendencia de Control del Poder de Mercado (2017). Indicadores de Diagnóstico Sectorial de la Competencia. Disponible en: http://www.scpm. gob.ec/indicadores-de-diagnostico-sectorial-de-la-competencia/. [Marzo 03, 2017]

Tirole, J. (1998). Theory of Industrial Organization. USA: Hamilton Printing. Tarziján, J., y Paredes, R. (2006). Organización industrial para la estrategia empresarial (Vol. 2). Pearson Educación.

Thong, T. Q. (2012). The SCP Paradigm-The Case Of Vietnam State-Owned Banks (No. 25).

U.S Department of Justice and the Federal Trade Commission. (2010). Horizontal Merger Guidelines. Washington, US: United States: Department of Justice and the Federal Trade Commission.

Vázquez, R. (2015). Concentración empresarial y cambio estructural: alimentos, bebidas y tabaco en México. Problemas del desarrollo. 46(180), 51-76.

\section{Agradecimientos:}

Este trabajo ha sido realizado en colaboración de la Universidad Metropolitana del Ecuador, Sede Machala y con el apoyo y asesoramiento de la Superintendencia de Compañías, Valores y Seguros del Ecuador.

Todos los comentarios son de exclusiva responsabilidad de los autores y no muestran la posición de la Superintendencia de Compañías, Valores y Seguros. 